\title{
DYNAMICAL SYSTEMS ON HOMOGENEOUS SPACES ${ }^{1}$
}

\author{
BY S. G. DANI
}

\author{
Communicated by J. A. Wolf, July 6, 1976
}

Consider the following class of dynamical systems: Let $X=G / \Gamma$ be the homogeneous space of a Lie group $G$ where $\Gamma$ is a lattice; i.e. a discrete subgroup of $G$ such that $G / \Gamma$ carries a finite $G$-invariant Borel measure $\mu$. Let $T$ be an affine transformation (AT) of $G / \Gamma$ i.e. $T=T_{g} \circ \bar{A}$ where $\bar{A}$ is the quotient of a continuous group automorphism $A$ of $G$ such that $A \Gamma \subset \Gamma$ and $T_{g}$ is the (left) translation of $G / \Gamma$ by an element $g \in G$. If the differential $d A$ of the automorphism is of determinant \pm 1 then $T$ is a measure-preserving transformation (MPT) of $X$; i.e. a dynamical system. More generally one may also consider groups of AT's, a typical case being a group of translation (i.e. $\bar{A}=\mathrm{Id}$ ) defined by a subgroup $H$ of $G$. These systems together with their factors include many classical dynamical systems, e.g. toral automorphisms, geodesic and horocycle flows on a surface of constant negative curvature and, more generally, the $G$-induced Anosov systems on infra-homogeneous spaces (cf. [7]).

The purpose of this note is to announce various ergodic and other dynamical properties of AT's, and more generally of groups of AT's, and discuss their applications. Analogues of these results have also been proved for the case when $G$ is the group of $k$-rational points of an algebraic group defined over a nondiscrete locally compact field $k$. However for simplicity here we confine ourselves to homogeneous spaces of Lie groups. The general statements as well as proofs of the results will appear elsewhere [2].

1. Bernoulli shifts. We have the following theorem.

Theorem 1. Let $T=T_{g} \circ \bar{A}$ be an $A T$ of $G / \Gamma$ and $\sigma=\operatorname{Ad} g \circ d A$ be the induced automorphism of the Lie algebra $g$ of $G$ (Ad denotes the adjoint action of $G$ on $\mathrm{g})$. Assume that

(i) the restriction of $\sigma$ to the maximal $\sigma$-invariant subspace on which all eigenvalues (possibly complex) are of unit absolute value, is a semisimple linear transformation and

(ii) $T$ is a Kolmogorov automorphism.

Then $T$ is a Bernoulli shift.

AMS (MOS) subject classifications (1970). Primary 54H20, 28A65, 58F99; Secondary $22 \mathrm{E} 40$.

${ }^{1}$ Details of proofs will appear in the Journal of the Indian Mathematical Society. 
It may be worth mentioning that as in [4], we prove every sufficiently smooth partition to be very weakly Bernoullian (cf. [4]).

For various classes of groups, conditions for an AT to be a Kolmogorov automorphism are known (cf. [5] and [1]). Thus using Theorem 1 we get algebraic (sufficient) conditions for an AT to be a Bernoulli shift (BS). In particular using results in [1] and Theorem 1 we conclude that translation by a semisimple element $g$ (i.e. Ad $g$ is a semisimple linear automorphism) is a BS whenever it is ergodic. This result, in particular, implies existence of vector groups of any dimension of MPT's such that every nontrivial MPT in the group is a BS, thus generalizing existence of a Bernoulli flow.

We also conclude from Theorem 1 that $G$-induced Anosov diffeomorphisms on infra-nilmanifolds and weakly mixing $G$-induced Anosov flows on infrahomogeneous spaces (cf. [7]) are Bernoullian.

2. Horospherical foliations. The horospherical foliations of an AT can be easily seen to be the foliations of $G / \Gamma$ into orbits of certain groups which we call the horospherical subgroups (HS) associated to the AT.

THEOREM 2. If $T$ is a weakly mixing $A T$ satisfying condition (i) in Theorem 1 , and $G / \Gamma$ is compact then every orbit of an HS associated to $T$ is dense in $G / \Gamma$.

The theorem is used to conclude minimality of actions of certain unipotent subgroups (e.g. maximal unipotent subgroups in $G$ ) on $G / \Gamma$. In particular we are able to deduce Greenberg's result (cf. [3]) on minimality of actions of lattices. The theorem also clearly implies density of each leaf of the horospherical foliations of any factor (topological) of an AT and, in particular, may be applied to deduce that each leaf of the horospherical foliations of a $G$-induced Anosov diffeomorphism or a $G$-induced weakly mixing Anosov flow is dense.

3. Generalised horocycles. Let $G$ be a semisimple Lie group without compact factors. It turns out that $g \in G$ belongs to a horospherical subgroup of an $\mathrm{AT}$ of $G / \Gamma$ (irrespective of $\Gamma$ ) only if $g$ is unipotent (i.e. all eigenvalues of Ad $g$ are 1). Further, whenever a translation by a nontrivial unipotent element which we call a generalised horocycle (GH) is ergodic, it has Lebesgue spectrum of infinite multiplicity and its entropy is zero. However, using Kirilov's notion of sequential entropy we prove the following result.

THEOREM 3. There are infinitely many nonisomorphic ergodic generalised horocycles, each modelled over a homogeneous space of a simple Lie group.

4. Anosov-like $n$-parameter groups. The construction of $G$-induced Anosov flow on group of (real) rank 1 can be (in a natural way) generalised to get $n$-parameter (Anosov-like) groups on infra-homogeneous spaces of rank $n$. Also appropriate analogues of ergodic and other dynamical properties (e.g. mixing, 
Kolmogorov property, density of leaves of horospherical foliations, etc.) of Anosov flows turn out to be valid for any rank. Lastly by reinterpreting a result of [6] we also conclude that the set of periodic points (i.e. points on compact orbits) is dense.

\section{REFERENCES}

1. S. G. Dani, Kolmogorov automorphisms on homogeneous spaces, Amer. J. Math. 98 (1976), 119-163.

2. - Bernoullian translations and minimal horospheres on homogeneous spaces, J. Indian Math. Soc. (to appear).

3. L. Greenberg, Discrete groups with dense orbits: Flows on homogeneous spaces, Ann. of Math. Studies, no. 53, pp. 85-103.

4. D. S. Ornstein and B. Weiss, Geodesic flows are Bernoullian, Israel J. Math. 14 (1973), 184-198. MR 48 \#4272.

5. W. Parry, Ergodic properties of affine transformations and flows on nilmanifolds, Amer. J. Math. 91 (1969), 757-771. MR 41 \#5595.

6. Gopal Prasad and M. S. Raghunathan, Cartan subgroups and lattices in semi-simple groups, Ann. of Math. (2) 96 (1972), 296-317. MR 46 \#1965.

7. Per Tomter, Anosov flows on infra-homogeneous spaces, Proc. Sympos. Pure Math., vol. 14, Amer. Math. Soc. Providence, R.I., 1970, pp. 299-327. MR 43 \#5552.

DEPARTMENT OF MATHEMATICS, TATA INSTITUTE OF FUNDAMENTAL RESEARCH, BOMBAY, INDIA 